\title{
A quantitative model for cyclotron wave-particle interactions at the plasmapause
}

\author{
D. L. Pasmanik ${ }^{1}$, V. Y. Trakhtengerts ${ }^{1}$, A. G. Demekhov ${ }^{1}$, A. A. Lyubchich ${ }^{2}$, E. E. Titova ${ }^{2}$, T. Yahnina ${ }^{2}$, \\ M. J. Rycroft ${ }^{3}$, J. Manninen ${ }^{4}$, T. Turunen ${ }^{4}$ \\ 1 Institute of Applied Physics, Nizhny Novgorod, Russia \\ 2 Polar Geophysical Institute, Apatity, Russia \\ 3 International Space University, Illkirch, France \\ ${ }^{4}$ Geophysical Observatory, Sodankylä, Finland
}

Received: 6 January 1997 / Revised: 3 September 1997 / Accepted: 12 September 1997

\begin{abstract}
The formation of a zone of energetic electron precipitation by the plasmapause, a region of enhanced plasma density, following energetic particle injection during a magnetic storm, is analyzed. Such a region can also be formed by detached cold plasma clouds appearing in the outer magnetosphere by restructuring of the plasmasphere during a magnetic storm. As a mechanism of precipitation, wave-particle interactions by the cyclotron instability between whistler-mode waves and electrons are considered. In the framework of the selfconsistent equations of quasi-linear plasma theory, the distribution function of trapped electrons and the electron precipitation pattern are found. The theoretical results are compared with experimental data obtained from NOAA satellites.
\end{abstract}

Key words. Magnetospheric physics · Energetic particles $\cdot$ Precipitating and trapped - Plasma waves and instabilities

\section{Introduction}

Many data on energetic particles in the magnetosphere have been obtained using high and low Earth-orbiting satellites. These data include the differential fluxes of radiation belt electrons and ions in different energy intervals, particle precipitation patterns, the characteristics of accompanying electromagnetic ELF-VLF emissions and cold plasma measurements. Satellite data are complemented by rich information about ELF-VLF emissions recorded at ground-based stations.
These data reveal some definite, regular features. In particular, the systematic precipitation of energetic electrons, which is correlated with ELF-VLF chorus generation, is seen on the morning side of the magnetosphere $(3<L<6)$. Also, a precipitation zone of energetic electrons has been observed on the evening/ afternoon side after a sufficiently strong magnetic storm. This zone has some remarkable features, investigated by Yahnina et al. (1996) and Titova et al. (1997) using the data of low-altitude $\left(h \sim 10^{3} \mathrm{~km}\right)$ NOAA satellites. These features include steady-state and spatially localized distributions of precipitated and trapped electrons, which have a specific cliff-like form along the satellite trajectory correlated with ELF emissions and with the locations of a sharp enhancement of background plasma density.

It is generally accepted that energetic electron precipitation which is correlated with strong ELF-VLF emissions is due to cyclotron wave-particle interactions inside the plasmapause or detached plasma regions with enhanced cold plasma density (Bespalov and Trakhtengerts, 1986). Quantitative interpretation of energeticelectron data demands the development of nonlinear models of such cyclotron wave-particle interactions, including real sources and sinks of both particles and waves in the magnetosphere. Attempts to construct such a model were first made for the electron radiation belts by Bespalov and Trakhtengerts (1986) and, similarly, for the ion ring current by Bespalov et al. (1990, 1994), in the frame of quasi-linear theory. These models lead to a very useful picture of the turbulent losses of energetic particles, and their dependence on plasmaspheric structure and on the character of the particle source. However, their application to the interpretation of particular experimental data, such as NOAA satellite data, requires a joint consideration of weak and strong pitch-angle diffusion.

The aim of this paper is to construct, in the framework of quasi-linear theory, a model of cyclotron wave-particle interactions, including real sources and sinks of particles and waves, and different regimes of 
pitch-angle diffusion. In particular, this model describes cyclotron wave-particle interactions during magnetic storms, when intense injection events take place on the nightside of the magnetosphere, and turbulent losses occur in the region where the injected particles encounter the plasmapause during their magnetic and electric drifts in longitude. Preliminary results of this study have been reported by Trakhtengerts et al. (1996). This paper is devoted to a more rigorous treatment of the stormrelated precipitation at the plasmapause for different regimes of pitch angle diffusion. Section 2 contains a mathematical formulation of the problem, and the solution is analysed in Sect. 3. A discussion of the results and a comparison with experimental data are given in Sect. 4. The conclusion in Sect. 5 contains some summarizing comments and recommendations for future investigations.

\section{Basic equations}

The developed model can be used to explain the properties of radiation belt electrons and ions. Here we shall deal with electron precipitation, bearing in mind the NOAA energetic-electron data mentioned in the introduction. We consider that a zone of electron precipitation is formed when energetic electrons interact via the whistler-wave cyclotron instability in a region of relatively dense cold plasma. This region may be at the plasmapause or a detached region caused by a restructuring of the plasmasphere during a magnetic storm. The source of energetic electrons is due to a substorm on the nightside, they enter the interaction region by longitudinal drift. We also assume that the NOAA data demonstrate steady-state features, allowing us to develop a time-stationary model. It is not always possible to distinguish temporal and spatial variations in experimental data, but the recurrence of specific features on different satellite passes, such as cliff width, height and location, is likely to indicate a time-stationary behaviour.

The quasi-linear self-consistent set of equations is used, including the diffusive equation for the distribution function $F$ and the wave energy transfer equation for the spectral density $\varepsilon_{\omega}$ of the whistler-mode waves, which are averaged over the bounce oscillations of electrons between magnetic mirror points and wavepackets between conjugate ionospheres. In our case of interest, most of the wave energy density occurs at low frequencies $\omega \ll \omega_{B L}$ (where $\omega_{B L}$ is the electron gyrofrequency in the equatorial plane), pitch-angle diffusion prevails, and the basic equations are written in the form (Bespalov and Trakhtengerts, 1986):

$$
\begin{aligned}
& \Omega_{D} \frac{\partial F}{\partial \varphi}=\frac{1}{T_{b}} \frac{\partial}{\partial \mu} \mu D \frac{\partial F}{\partial \mu}-\delta \cdot F, \\
& v_{g \perp} \frac{\partial \varepsilon_{\omega}}{\partial r_{\perp}}=(\gamma-v) \varepsilon_{\omega},
\end{aligned}
$$

where the left term in Eq. (1) describes the longitudinal magnetic drift, $\varphi$ is the azimuthal angle, $\Omega_{D}$ is the angular drift velocity, $T_{b}$ is the electron bounce period $T_{b}=\oint d z / v_{\|}, v_{\|}$is the velocity component parallel to the Earth magnetic field, $z$ is the coordinate along the magnetic field line, the magnetic moment $\mu=\sin ^{2} \Theta_{L}$ and $\Theta_{L}$ is the pitch-angle in the equatorial plane for a certain $L$ value; the last term in Eq. (1) characterizes the particle losses via pitch angle diffusion (coefficient $D$ ) through the loss cone, with the coefficient $\delta$ being equal to

$\delta=\left\{\begin{array}{ll}0 & \mu \geq \mu_{c}, \\ \delta_{0}=v / l & 0 \leq \mu \leq \mu_{c}\end{array}\right.$,

where $\mu_{c}=L^{-3}\left(4-3 L^{-1}\right)^{-1 / 2}$ is the edge of the loss cone for a dipolar magnetic field, $v$ is the electron velocity and $l$ is the length of the magnetic flux tube (between the conjugate ionospheres). The diffusion coefficient $D$ is determined by the wave intensity $\varepsilon_{\omega}$ and depends in general on $\mu$ and $v$; it is equal to

$D=\int_{\omega_{0}}^{\omega_{B L}} G \varepsilon_{\omega} d \omega$

where $\omega_{0}$ and $G$ are rather complicated functions of $\mu$ and $v$ (Bespalov and Trakhtengerts, 1986). These dependencies are not crucial for the qualitative solution of Eqs. (1) and (2) if the number of non-resonant electrons is small. Such a situation occurs in the case of a sharp and dense plasmapause, when the cyclotron resonance condition in the equatorial plane

$\omega-\omega_{B L}=k_{L} v_{\| L}$

is satisfied for practically all energetic electrons. Here $k_{L} \sim n_{c}^{1 / 2}$ is the wave vector of whistler waves, and $n_{c}$ is the cold plasma density. Oblique whistler waves with $\mathbf{k} \nvdash \mathbf{B}$, which appear at the generation region due to wave refraction, smooth the dependence of $D$ on $\mu$ and $v$. Taking into account these circumstances, we shall suppose further that $D$ [Eq. (4)] does not depend on $\mu$, that is

$D=V\left(v^{2}\right) \tilde{D}=V \cdot \int_{\omega_{0}}^{\omega_{B L}} G_{1} \varepsilon_{\omega} d \omega$,

where, according to Bespalov and Trakhtengerts (1986),

$V=\left(\frac{4 \pi e}{m c v}\right)^{2}, \quad G_{1}=\frac{l_{\text {eff }}}{k}$

$l_{\text {eff }}$ is the effective length of the whistler-electron interaction region near the equator at a certain $L$ value. Here $e$ is the charge of the electron of mass $m$, and $c$ is the velocity of light.

The energy transfer equation [Eq. (2)] for whistler waves includes the energy source, which is described by the term $\gamma \varepsilon_{\omega} ; \gamma$ is the growth rate due to the cyclotron instability. The energy losses are due to two processes: the imperfect reflection from the ionosphere (the term $v \varepsilon_{\omega}$, where $v$ is the damping rate) and propagation away from the generation region across the magnetic field due to refraction (the term $v_{g \perp} \frac{\partial \varepsilon_{\omega}}{\partial r_{\perp}}$ ). In Eq. (2), $v_{g \perp}$ and $r_{\perp}$ are 
the component of whistler-mode group velocity and spatial coordinate perpendicular to the magnetic field, respectively. The main propagation term $v_{g z} \frac{\partial \varepsilon_{\omega}}{\partial z}$ has been removed by averaging over the oscillations of wavepackets between conjugate ionospheres. Further, we consider a one-dimensional problem and put $d r_{\perp}=R_{0} L d \varphi$, where $R_{0}$ is the Earth radius.

It should be mentioned that we do not consider the strict model for whistler wave propagation near the plasmapause, which serves as a waveguide [see, for example, Semenova and Trakhtengerts (1980), Strangeways (1991)]. This is an important problem, which includes the analysis of the eigenmodes' spatial structure as well as the refraction of group rays across plasmapause waveguide, and demands special consideration. The last effect is taken into account in Eq. (2) by the model term $v_{g \perp} \partial \varepsilon_{\omega} / \partial r_{\perp}$, with $v_{g \perp}$ as the parameter.

The growth rate $\gamma$ can be written (Bespalov and Trakhtengerts, 1986) as:

$\gamma \approx \frac{1}{T_{g}} \frac{(2 \pi)^{3} e}{c B_{L}} \int_{\omega_{B L} / k}^{\infty} \int_{0}^{\mu_{m}} l_{\mathrm{eff}} \mu v^{3}\left(\frac{\partial F}{\partial \mu}-\frac{\omega}{\omega_{B L}} F\right) \mathrm{d} \mu \mathrm{d} v$

where $\mu_{m}=1-\left(\omega_{B L} / k v\right)^{2}, \quad T_{g}=\oint d z / v_{g} \|$ is time of wavepacket oscillations between conjugate ionospheres, and the electron distribution function is normalized by the relation:

$N=\pi \mu_{c}^{-1} \int_{0}^{\infty} \int_{0}^{1} T_{b} F v^{3} \mathrm{~d} \mu \mathrm{d} v$

$N$ is the number of electrons in the magnetic flux tube at a certain $L$ value with unit cross-section in the ionosphere.

The self-consistent system of Eq. (1) and (2) must be completed by the initial (on the $\varphi$-axis) and boundary (over $\mu$ ) conditions, which have the form:

$\varphi=\varphi_{0}: \varepsilon_{\omega}=\varepsilon_{\omega 0}, F=F_{0}(\mu)$,

$\mu=0 ; 1: \mu \frac{\partial F}{\partial \mu}=0$.

Here $\varphi_{0}$ is the longitude (local time) where drifting energetic electrons meet the sharp cold plasma density enhancement; the boundary conditions over $\mu$ are that the diffusion flow is equal to zero at the limits of $\mu$.

\section{Solution}

The solution of Eqs. (1) and (2) is rather difficult, in spite of the equations' simple form. We shall select two cases. The first is the case of strong pitch-angle diffusion (SD), when the time of loss-cone filling in the process of pitchangle diffusion $\left(D / \mu_{c} T_{b}\right)^{-1}$ is much less than the time required to empty the loss cone, $\delta_{0}^{-1}$. Here $\mu_{c}$ is the magnetic moment at the edge of the loss cone. This condition means that the loss cone is constantly filled. In the case when the distribution function is close to isotropic, we can consider Eq. (1) averaged over $\mu$, where the term characterizing the total particle losses can be written as $\delta_{0} \mu_{c} F$. Then the solution of Eq. (1) has the form

$F=\tilde{F} \cdot \exp (-\Delta \cdot \varphi)$,

where $\Delta=\delta_{0} \mu_{c} / \Omega_{D}$ and $\tilde{F}$ is the average value of the initial distribution function $F_{0}(\mu)$. This approximation cannot be used at the stage of isotropization, when the distribution function is sufficiently anisotropic. Nevertheless, at this stage the solution of Eq. (1) can be approximated by the solution of this equation with $\delta \equiv 0$ and losses taken into account as the exponential multiplier, Eq. (11), to the isotropic part of the distribution function (zero eigenfunction with $v_{0}=0$, see the following).

The criteria of strong diffusion can be written in the form (Kennel, 1969; Bespalov and Trakhtengerts, 1986):

$K \equiv \frac{D}{\mu_{c} T_{b} \delta_{0}} \approx \frac{D}{1.5 \mu_{c}} \gg 1$,

where we have used the approximate relation of Lyons and Williams (1984): $T_{b}(\mu=0) /\left\langle T_{b}\right\rangle \approx 1.3$.

In the opposite case

$K \ll 1$

we deal with weak diffusion (WD), when the loss cone is empty, and it is possible to consider only the solution for $\mu \geq \mu_{c}$ with the additional boundary condition under $\mu=\mu_{c}$,

$F\left(\mu=\mu_{c}, \varphi\right)=0$.

So, in both cases, Eq. (1) is reduced to

$\frac{\partial F}{\partial \xi}=\frac{\partial}{\partial \mu} \mu \frac{\partial F}{\partial \mu}$

but with different boundary conditions in the case of strong and weak diffusion (SD and WD, respectively). A new variable $\xi$ is defined as

$d \xi=\frac{D}{T_{b} \Omega_{D}} \mathrm{~d} \varphi$

This actually means a rescaling of the longitudinal coordinate, which depends now on the wave intensity. This allows us to solve the equation for the distribution function $F(\xi, \mu)$ independently of Eq. (2). The dependence $\xi(\varphi)$ can be found after Eq. (2) is analysed (see the following).

Further, we assume that the distribution function of energetic electrons has a small spread over energies: $\Delta v / v_{0} \ll 1$, where $v_{0}$ and $\Delta v$ are the characteristic velocity and dispersion of the distribution function of energetic electrons, respectively. In this approach the following form of the distribution function can be used:

$F=\frac{1}{2 \pi v_{0}^{3}} \delta\left(v-v_{0}\right) \mathscr{F}(\varphi, \mu)$

In fact, all the results discussed in the following can be obtained for any spread of the distribution over energy, but the solution will have a very complicated form; the 
qualitative picture is the same, but a detailed comparision of the theoretical and experimental results will be more difficult.

By using a Laplace transformation on $\xi$ and then solving the resulting Sturm-Liouville problem in $\mu$, the solution of Eq. (15) can be written as a series in terms of the eigenfunctions of the diffusion operator [see, for example, Korn and Korn (1961)]:

$$
\mathscr{F}=\sum_{k} A_{k} Z_{k}(\mu) \exp \left(-v_{k} \xi\right) \text {, }
$$

where $Z_{k}$ and $v_{k}$ are eigenfunctions and eigenvalues; the coefficient $A_{k}$ is given by:

$A_{k}=\left(\int_{0}^{1} \mathscr{F}_{0}(\mu) Z_{k}(\mu) \mathrm{d} \mu\right)\left(\int_{0}^{1} Z_{k}^{2}(\mu) \mathrm{d} \mu\right)^{-1}$.

In the SD case the full solution is given by Eq. (11) and the eigenfunctions

$Z_{k}^{S}(\mu)=J_{0}\left(2 \sqrt{v_{k} \mu}\right), \quad k=0,1, \ldots$,

where the eigenvalues $v_{k}$ are the roots of the equation

$J_{1}\left(2 \sqrt{v_{k} \mu}\right)=0$.

In the WD case

$Z_{k}^{W}(\mu)=J_{0}\left(2 \sqrt{p_{k} \mu}\right)-\frac{J_{0}\left(2 \sqrt{p_{k} \mu_{c}}\right)}{N_{0}\left(2 \sqrt{p_{k} \mu_{c}}\right)} N_{0}\left(2 \sqrt{p_{k} \mu}\right)$

$k=1,2, \ldots$

where, unlike the SD case, the eigenvalues are defined as $p_{k}$. They are found from the characteristic equation

$$
\begin{aligned}
& J_{1}\left(2 \sqrt{p_{k}}\right) N_{0}\left(2 \sqrt{p_{k} \mu_{c}}\right) \\
& \quad-N_{1}\left(2 \sqrt{p_{k}}\right) J_{0}\left(2 \sqrt{p_{k} \mu_{c}}\right)=0,
\end{aligned}
$$

where $J_{0,1}$ and $N_{0,1}$ are the Bessel functions of the first and second kinds, respectively.

Different sets of eigenvalues and eigenfunctions in the cases of strong and weak diffusion lead to a considerable difference in the behaviour of the solution. In the SD case, the zero eigenvalue exists $\left(v_{k}=0\right)$ which corresponds to strong isotropization of the distribution function with an increase of $\xi$. Its amplitude decreases in correspondence with Eq. (11) due to the exponential multiplier $\exp (-\Delta \cdot \varphi)$. In the WD case, an isotropic component is absent, and the distribution function quickly approaches the eigenfunction $Z_{k}^{W}$ with the minimal value of $p_{k}$, as $\xi$ increases.

Substituting Eq. (18) into Eq. (8), it is possible to find the dependence $\gamma(\xi, \omega)$. Then, from Eq. (2), we can obtain an integro-differential equation for the spectral density $\varepsilon_{\omega}$, which can be used to perform the analysis of wave spectra. This analysis is rather complicated and will be performed in another paper together with the spread of energetic electrons over energy being taken into account. Here our interest is to find the fluxes of trapped and precipitated electrons, which depend on the wave intensity integrated over all frequencies. To find this value we use the fact that the increment $(\gamma-v)$ has a maximum as a function of frequency at the point $\omega=\omega_{m} \ll \omega_{B L}$

For a quasi-steady process the wave spectral intensity $\varepsilon_{\omega}$ will have a sharp maximum near the same frequency $\omega_{m}$. Hence, it is possible to obtain from Eqs. (2) and (4) the equation for the diffusion coefficient $D$, integrating both parts of Eq. (2) over $\omega$ with the weighting function $G_{1}$. The result is

$\frac{\tilde{v}_{g \perp}}{R_{0} L} \frac{\partial D}{\partial \varphi}=\left(\gamma_{m}-v_{0}\right) D$

where $\tilde{v}_{g \perp}, \gamma_{m}$ and $v_{0}$ are the values corresponding to $\omega=\omega_{m}$, and

$\gamma_{m}=\gamma_{0} \int_{0}^{1} \mu \frac{\partial \mathscr{F}}{\partial \mu} \mathrm{d} \mu$

where

$\gamma_{0}=\pi \frac{l_{\text {eff }}}{n_{c L} l} k\left(\omega_{m}\right)$.

Here $n_{c L}$ is the plasma density in the equatorial plane for a certain $L$ value.

Applying Eq. (16) to Eq. (24) we obtain

$\frac{\partial D}{\partial \xi}=\alpha\left(\gamma_{m}-v_{0}\right)$,

where the coefficient $\alpha$ is equal to:

$\alpha=R_{0} L T_{b} \Omega_{D} / \tilde{v}_{g \perp}$

The growth rate $\gamma_{m}$ is found from Eq. (25), using Eq. (18):

$\gamma_{m}=\sum_{k=1}^{\infty} A_{k} \gamma_{0}\left[\int_{0}^{1} \frac{\partial Z_{k}}{\partial \mu} \mu \mathrm{d} \mu\right] \cdot \exp \left(-\psi_{k}(\xi)\right)$,

where $\psi_{k}=v_{k} \xi$ in the case of SD, and $\psi_{k}=p_{k} \xi$, for WD. To simplify further calculations we note that the eigenvalues $v_{k}$ or $p_{k}$ grow rapidly with a number $k$, so only terms with minimal eigenvalue give a significant contribution to $\gamma$ when $\xi$ is growing. In the case of SD that is $v_{k}=0$ and $v_{k}=v_{1}$. In the case of WD, $\left(p_{k}\right)_{\min }=p_{1}$ is the first root of Eq. (23). Thus, we can take

$\gamma_{m}^{S} \approx B_{1}^{S} \exp \left(-v_{1} \xi\right)$

for SD (superscript $S$ ) and

$\gamma_{m}^{W} \approx B_{1}^{W} \exp \left(-p_{1} \xi\right)$

for WD (superscript $W$ ), where

$B_{k}=\gamma_{0} A_{k} \int_{0}^{1} \frac{\partial Z_{k}}{\partial \mu} \mu \mathrm{d} \mu, \quad k=1,2, \ldots$.

We can see that the dependencies of $\gamma_{m}^{S}$ and $\gamma_{m}^{W}$ on $\xi$ are the same, so we have a common equation for finding the function $\xi(\varphi)$, which is valid for both SD and WD regimes, and which has the form 
$\frac{d D_{S}}{d \xi}=\alpha\left(B_{1}^{S} \exp \left(-v_{1} \xi\right)-v_{0}\right)$

for the SD regime; for the WD regime it is necessary to replace $B_{1}^{S}$ and $v_{1}$ with $B_{1}^{W}$ and $p_{1}$. The solution of Eq. (33) is written as

$D \equiv T_{b} \Omega_{D} \frac{d \xi}{d \varphi} \approx D_{0}+\alpha \frac{B_{1}^{S}}{v_{1}}\left(1-e^{-v_{1} \xi}\right)-\alpha v_{0} \xi$

where

$D_{0}=\int_{\omega_{0}}^{\omega_{B L}} G \varepsilon_{\omega 0} d \omega$.

We shall suppose further that for the initial state of the instability $(\xi \rightarrow 0)$, the growth rate is much larger than the damping rate $v_{0}$, and the initial value of the diffusion coefficient $D_{0}$ is much less than the maximum value of $D_{m}$ :

$B_{1}^{S, W} \gg v_{0}, \quad D_{m} \gg D_{0}$.

In these conditions the value of $D_{m}$ and its position on the $\xi$ axis are

$D_{m} \approx \frac{\alpha B_{1}^{S}}{v_{1}}, \quad \xi_{m} \approx \frac{1}{v_{1}} \ln \frac{B_{1}^{S}}{v_{0}}$.

It is possible to find from the condition $D \geq 0$ the limits of $\xi$ :

$0 \leq \xi \leq \xi_{\infty}, \quad \xi_{\infty} \simeq \frac{B_{1}^{S}}{v_{0} v_{1}}$.

We find the dependence $\varphi(\xi)$ by integrating Eq. (34). With Eq. (36) taken into account, this dependence has the form:

$\varphi=\frac{\tilde{v}_{g \perp}}{R_{0} L B_{1}} \Phi(\xi)$

where

$\Phi= \begin{cases}\Phi_{1}, & 0 \leq \xi \leq \xi_{m}, \\ \Phi_{1}\left(\xi_{m}\right)+\Phi_{2}, & \xi_{m} \leq \xi \leq \xi_{\infty},\end{cases}$

$\Phi_{1}=\ln \left[\frac{D_{m}}{D_{0}}\left(\exp \left(v_{1} \xi\right)-1\right)+1\right]$,

$\Phi_{2}=\frac{B_{1}}{v_{0}} \ln \left[\frac{1-\xi_{m} / \xi_{\infty}}{1-\xi / \xi_{\infty}}\right] ;$

$D_{m}, \xi_{m}$ and $\xi_{\infty}$ are determined by Eqs. (37)-(38). The substitution of Eq. (37) into Eq. (39) gives:

$\varphi_{m}=\frac{\tilde{v}_{g \perp}}{R_{0} L B_{1}} \ln \left(\frac{D_{m} B_{1}}{D_{0} v_{0}}\right)$.

\section{Discussion}

Discussion of the theoretical results centres on a comparison of the spatial behaviour of the fluxes of trapped and precipitated electrons, which comes from these calculations, with those derived from the analysis of experimental data. In particular, we consider NOAA satellite data on energetic electrons in the local evening hours (Yahnina et al., 1996).

The general expression for the electron flux $S$ through the unit cross-section of a magnetic flux tube is determined by the relation (Bespalov and Trakhtengerts, 1986)

$S=2 \pi b \int_{0}^{\infty} v^{3} \mathrm{~d} v \int_{0}^{1 / b} F(\mu, v) \mathrm{d} \mu$,

where the parameter $b(z)=B(z) / B_{L}$, and $z$ is the coordinate along the magnetic flux tube measured from the equator: $B(z=0)=B_{L}$. The flux $S_{p r}$ within the loss cone $0 \leq \mu \leq \mu_{c}$ corresponds to precipitating electrons. For the magnetosphere $\mu_{c} \ll 1$, and it is possible to write for the $\mathrm{SD}$ regime, $F(\mu) \approx F\left(\mu_{c}\right) \approx$ constant, so

$S_{p r}^{S}=2 \pi \int_{0}^{\infty} v^{3} \mathrm{~d} v F_{S}\left(\mu_{c}, v\right)$.

For the WD regime $S_{p r}$ can be found from Eq. (1) if we integrate both parts of it over the volume of the magnetic flux tube with unit cross-section at its feet (Bespalov and Trakhtengerts, 1986):

$S_{p r}^{W}=\pi \int_{0}^{\infty} v^{3} \mathrm{~d} v\left(D \frac{\partial F_{W}}{\partial \mu}\right)_{\mu_{c}}$.

The trapped electrons flux $S_{t r}$ for both regimes is defined as

$S_{t r}^{S, W}=2 \pi b \int_{0}^{\infty} v^{3} \mathrm{~d} v \int_{\mu_{c}}^{1 / b} F(\mu, v) \mathrm{d} \mu$.

Taking into account Eq. (17), Eqs. (45)-(47) can be written as

$S_{p r}^{S}(\xi)=\mathscr{F}_{S}\left(\mu_{c}, \xi\right)$

$S_{p r}^{W}(\xi)=\frac{1}{2}\left(D \frac{\partial \mathscr{F}_{W}}{\partial \mu}\right)_{\mu_{c}}$,

$S_{t r}^{S, W}(\xi)=b \int_{\mu_{c}}^{1 / b} \mathscr{F}(\mu, \xi) \mathrm{d} \mu$.

Qualitative pictures of the electron fluxes of trapped and precipitated particles at sufficiently low heights, which follow from the experimental data analysis (Yahnina et al., 1996), are presented in Fig. 1: part a corresponds to the SD case, and part $\mathbf{b}$ to the case of WD; the thick line illustrates the cold plasma density profile encountered by the longitudinally drifting energetic electrons. In both regimes at the initial stage the fluxes of trapped electrons have a very sharp increase accompanied by the appearance of the precipitated electrons flux. This feature was called a "cliff" by 


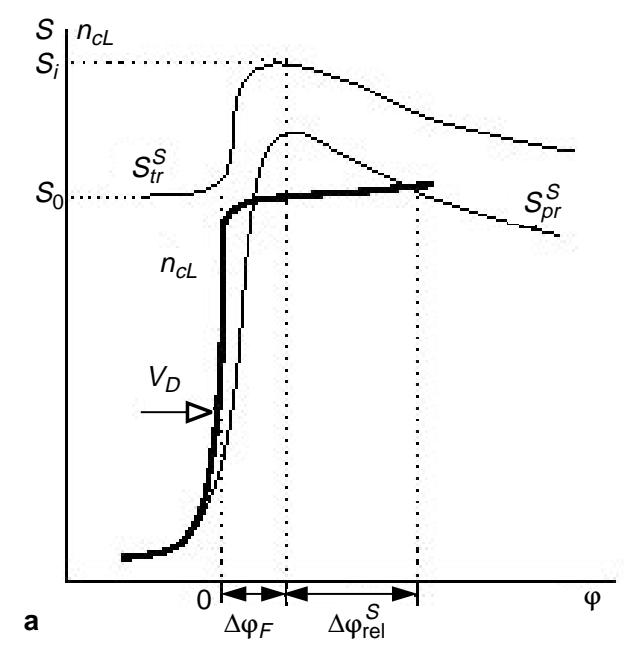

Yahnina et al. (1996). According to this picture we can take the following values as parameters of the "cliff": the fractional increase of trapped electrons flux $(q)$, the width of the front of the cliff $\left(\Delta \varphi_{F}\right)$, and the characteristic width of relaxation $\left(\Delta \varphi_{\text {rel }}\right)$.

To find these values in the framework of the developed model and compare them with experimental data, we need to know the initial distribution function of the energetic electrons $F_{0}=\left(2 \pi v_{0}^{3}\right)^{-1} \delta\left(v-v_{0}\right) \mathscr{F}_{0}$. In this work we use the following dependence for $\mathscr{F}_{0}(\mu)$ :

$$
\mathscr{F}_{0}(\mu)=\left\{\begin{array}{ll}
C_{\beta}\left(\mu-\mu_{c}\right)^{\beta}, & \mu>\mu_{c}, \\
0, & \mu<\mu_{c},
\end{array},\right.
$$

where $\beta>0$ is a number characterizing the anisotropy of the initial distribution function. This expression can be used as a good approximation of many different functions by choosing an appropriate value of $\beta$. The normalizing constant $C_{\beta}$ can be expressed from Eq. (44) through the initial energetic electron flux $S_{0 L}$ in the equatorial plane:

$C_{\beta} \simeq(1+\beta) S_{0 L}$.

Substituting Eq. (51) into Eq. (50) we find the distribution of the initial flux of trapped electrons $S_{t r}(z)$ along the magnetic flux tube:

$S_{t r 0}(z)=S_{0 L} b\left(\frac{1}{b}-\mu_{c}\right)^{1+\beta}$.

After isotropization, in the case of SD, the distribution function is equal to $\mathscr{F}(\mu)=A_{0} \exp (-\Delta \cdot \varphi)$, where according to Eq. (19) $A_{0}=C_{\beta} /(\beta+1)$, and the flux of trapped particles is

$S_{t r i}^{S}(z)=S_{0 L} b\left(\frac{1}{b}-\mu_{c}\right) \cdot \exp (-\Delta \cdot \varphi)$.

The ratio $\left(q^{S}\right)$ of flux after the isotropization to the initial flux gives us the relative height of the cliff of the flux density in the NOAA data, which is predicted by the theory:

$q^{S}=\left(\frac{S_{t r i}^{S}(z)}{S_{t r 0}(z)}\right)=\left(\frac{1}{b}-\mu_{c}\right)^{-\beta}$
Here we have neglected the exponential multiplier that is close to unity for corresponding values of $\varphi$.

The width of the front of the cliff is equal to $(\Delta \xi)_{F}^{S} \sim v_{1}^{-1}$ or, according to Eqs. (39)-(41),

$\Delta \varphi_{F}^{S} \sim\left(\frac{\tilde{v}_{g \perp}}{L R_{0} B_{1}^{S}}\right) \ln \left(\frac{D_{m}^{S}}{D_{0}}\right)$.

The characteristic width of the relaxation length is determined by the exponential multipliers Eq. (11), that gives

$\Delta \varphi_{r e l}^{S} \sim \Delta^{-1}=\frac{\Omega_{D}}{\delta_{o} \mu_{c}}$.

Putting $L=4.5, B_{1}=15 \mathrm{~s}^{-1}$ (this corresponds to the trapped electrons flux $S_{t r} \sim 10^{8} \mathrm{~cm}^{-2} \mathrm{~s}^{-1}$ of radiation belt electrons at the equatorial plane), $\tilde{v}_{g \perp}=10^{2} \mathrm{~km} / \mathrm{s}$ (which gives $D_{m} \mu_{c}^{-1} \sim 8$ ), and $\ln \left(D_{m} / D_{0}\right)=10$, we find that $\Delta \varphi_{F}^{S} \approx 0.12^{\circ}$ and $\Delta \varphi_{\text {rel }}^{S} \sim 0.25^{\circ}$.

For the case of WD, only partial isotropization takes place, when the initial function goes to the first eigenfunction $Z_{1}^{W}(\mu)$. Apparently, this occurs at the distance $(\Delta \xi)_{F}^{W} \sim p_{2}^{-1}$ from the point of energetic particle injection, where $p_{2}$ is the second eigenvalue. Thus, to estimate the value of $\Delta \varphi_{F}^{W}$ we should take into account two eigenfunctions in Eq. (33). Then it is possible to obtain the dependence $\varphi(\xi)$ similar to Eqs. (39)-(41) that gives us:

$\Delta \varphi_{F}^{W} \sim\left(\frac{\tilde{v}_{g \perp}}{L R_{0}\left(B_{1}^{W}+B_{2}^{W}\right)}\right) \ln \left(\frac{D_{m}^{W}}{D_{0}}\right)$,
$D_{m}^{W} \simeq \alpha\left(B_{1}^{W}+B_{2}^{W}\right)$.

This value is similar to the SD case of Eq. (56), and the initial distribution function determines the values of $B_{1,2}^{W}$ [Eq. (32)]. The relaxation in the WD regime is determined by the multiplier $\exp \left(-p_{1} \xi\right)$, so we have the characteristic scale of relaxation $\Delta \xi \sim p_{1}^{-1}$, or from Eqs. (39)-(41)

$\Delta \varphi_{r e l}^{W} \sim\left(\frac{\tilde{v}_{g \perp}}{L R_{0} B_{1}^{W}}\right) \ln \left(\frac{D_{m}^{W}}{D_{0}}\right)$. 
Putting $L=4.5, B_{1} \approx 2 B_{2}=0.3 \mathrm{~s}^{-1}$ (this corresponds to the trapped electrons flux $S_{t r} \sim 5 \cdot 10^{6} \mathrm{~cm}^{-2} \mathrm{~s}^{-1}$ of radiation belt electrons at the equatorial plane), $\tilde{v}_{g \perp}=10^{2} \mathrm{~km} / \mathrm{s} \quad$ (which gives $D_{m} \mu_{c}^{-1} \sim 0.3$ ), and $\ln \left(D_{m} / D_{0}\right)=10$, we find that $\Delta \varphi_{F}^{W} \approx 4.4^{\circ}$ and $\Delta \varphi_{\text {rel }}^{S} \approx 6.6^{\circ}$.

To estimate the height of the cliff in the WD case we consider that, at the distance $(\Delta \xi)_{F}^{W}$, only the first term of the series of Eq. (18) is sufficient. According to Eqs. (18) and (22):

$$
\begin{gathered}
\mathscr{F}^{W} \approx A_{1} \exp \left(-v_{k} \xi\right)\left\{J_{0}\left(2 \sqrt{p_{1} \mu}\right)\right. \\
\left.-Q N_{0}\left(2 \sqrt{p_{1} \mu}\right)\right\},
\end{gathered}
$$

where $Q=J_{0}\left(2 \sqrt{p_{1} \mu_{c}}\right) / N_{0}\left(2 \sqrt{p_{1} \mu_{c}}\right)$.

The normalizing constant $A_{1}$ can be determined using the fluxes in the equatorial plane:

$$
\begin{aligned}
A_{1} & =\left(\int_{\mu_{c}}^{1} \mathscr{F}_{0} Z_{1} d \mu\right)\left(\int_{\mu_{c}}^{1} Z_{1}^{2} d \mu\right)^{-1} \\
& =S_{0 L}(\beta+1)\left(\int_{\mu_{c}}^{1}\left(\mu-\mu_{c}\right)^{\beta} Z_{1} d \mu\right)\left(\int_{\mu_{c}}^{1} Z_{1}^{2} d \mu\right)^{-1} .
\end{aligned}
$$

We shall suppose that for the case of low-orbit satellite data the inequality is satisfied:

$2 \sqrt{p_{1} / b} \ll 1$

since a typical value of $p_{1} \sim 1$ and for a low-orbit satellite $b \sim 60$. In this approach

$Z_{1}^{W}(\mu) \approx \mathscr{Z}_{1}(\mu) \equiv 1-\frac{\ln \left(4 p_{1} \mu\right)}{\ln \left(4 p_{1} \mu_{c}\right)}$,

and the electron flux at any arbitrary point $z$ is equal to:

$S^{W}(z) \approx A_{1} b \mathrm{e}^{-p_{1}(\Delta \xi)_{F}^{W}} \int_{\mu_{c}}^{1 / b} \mathscr{Z}_{1}(\mu) \mathrm{d} \mu$.

Taking into account that $(\Delta \xi)_{F}^{W} \sim p_{2}^{-1}$ and the typical value of $p_{1} / p_{2}>10$, we can neglect the exponential multiplier in Eq. (64), and the height of the cliff in the WD regime can be derived as the ratio of Eq. (64) to Eq. (53):

$$
\begin{aligned}
q^{W} \approx & (\beta+1)\left(b^{-1}-\mu_{c}\right)^{-1-\beta} \int_{\mu_{c}}^{1 / b} \mathscr{Z}_{1}(\mu) d \mu \\
& \times \int_{\mu_{c}}^{1}\left(\mu-\mu_{c}\right)^{\beta} Z_{1} d \mu\left(\int_{\mu_{c}}^{1} Z_{1}^{2} d \mu\right)^{-1} .
\end{aligned}
$$

It is clear from Eqs. (55) and (65) that the height of the cliff in the trapped electron fluxes in both regimes is determined only by the anisotropy of the initial distribution function and the satellite altitude. However, the height of the cliff in the WD case is less than in the SD case. For $L=4, b=\mu_{c}^{-1} / 2 \approx 60$ (which corresponds to the satellite altitude $h \sim 10^{3} \mathrm{~km}$ ) and $\beta=1$, we find that $q^{S} \approx 110, q^{W} \approx 13$, so $q^{S} / q^{W} \approx 8$.

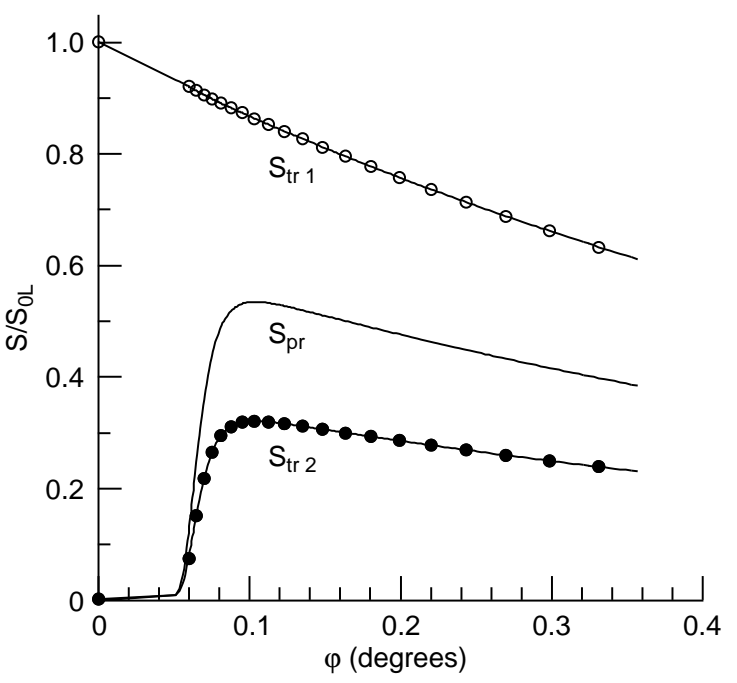

Fig. 2. The dependence of fluxes of trapped and precipitated electrons on $\varphi$ in the case of strong diffusion; $\mu_{c}^{-1}=160$, $D_{m} \mu_{c}^{-1} \approx 8 \quad\left(B_{1} \approx 15 \mathrm{~s}^{-1}, \quad\right.$ if $\left.v_{g \perp} \approx 10^{2} \mathrm{~km} / \mathrm{s}\right), \ln \left(D_{m} / D_{0}\right) \approx 10$, $v_{0} / B_{1}=0.01, S_{t r 1}=S_{t r}(b=1), S_{t r 2}=S_{t r}\left(b=\mu_{c}^{-1} / 1.6\right), b=B(z) / B_{L}$

Together with the analytical estimates, a computational analysis of the solutions of Eqs. (1)-(24) has been undertaken for the initial distribution function Eq. (51), with $\beta=1$. Examples of the fluxes of trapped and precipitated electrons are given in Figs. 2 and 3 as functions of $\varphi$ for the SD and WD regimes, respectively. It is seen that for the parameters chosen, the characteristic scales of growth and decay stages are comparable in the WD case, and that the WD decay stage is longer in comparison with the same scale in the SD case. Also, in the SD case the trapped and precipitated fluxes are comparable; however, for the WD case $S_{t r} \gg S_{p r}$. The dependencies of the trapped electron fluxes on $b(z)=B(z) / B_{L}$ for different values of $\varphi$ are given in

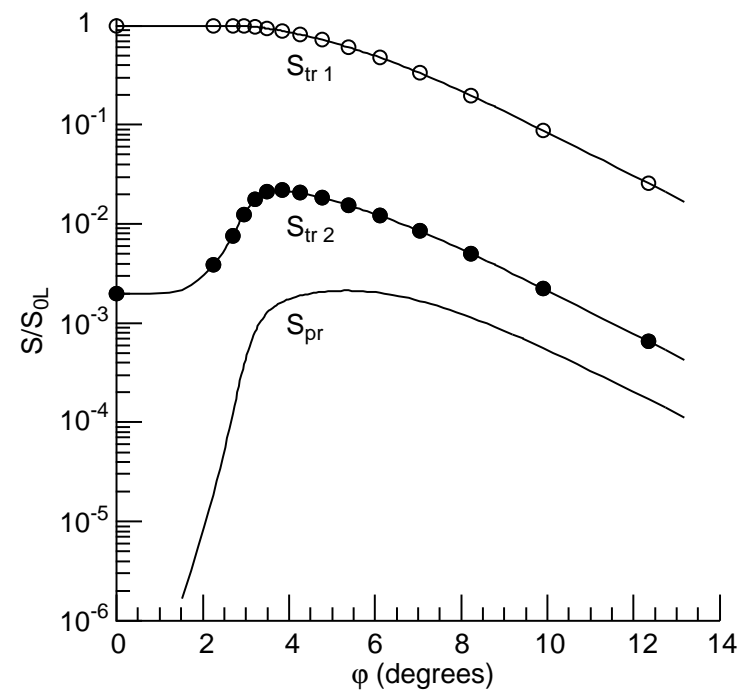

Fig. 3. The dependence of fluxes of trapped and precipitated electrons on $\varphi$ in the case of weak diffusion; $\mu_{c}^{-1}=160$, $D_{m} \mu_{c}^{-1} \approx 0.3\left(B_{1} \approx 0.5 \mathrm{~s}^{-1}\right.$, if $\left.v_{g \perp} \approx 10^{2} \mathrm{~km} / \mathrm{s}\right), \ln \left(D_{m} / D_{0}\right) \approx 10$, $v_{0} / B_{1}=0.01, S_{t r 1}=S_{t r}(b=1), S_{t r 2}=S_{t r}\left(b=\mu_{c}^{-1} / 1.6\right), b=B(z) / B_{L}$ 


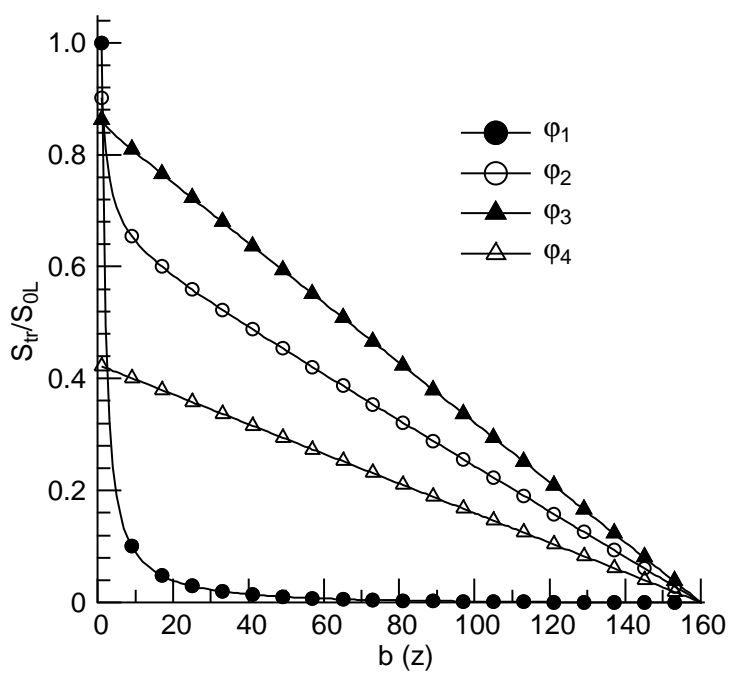

Fig. 4. The dependence of fluxes of trapped electrons along a magnetic flux tube on $b(z)=B(z) / B_{L}$, in the case of strong diffusion; $\mu_{c}^{-1}=160, \quad D_{m} \mu_{c}^{-1} \approx 8 \quad\left(B_{1} \approx 15 \mathrm{~s}^{-1}, \quad\right.$ if $\left.\quad v_{g \perp} \approx 10^{2} \mathrm{~km} / \mathrm{s}\right)$, $\ln \left(D_{m} / D_{0}\right) \approx 10, \quad v_{0} / B_{1}=0.01, \quad \varphi_{1}=0, \quad \varphi_{2} \approx 0.07^{\circ}, \quad \varphi_{3} \approx 0.1^{\circ}$, $\varphi_{4} \approx 0.62^{\circ}$

Figs. 4 and 5. The first curve on these figures $\left(\varphi_{1}=0\right)$ corresponds to the initial distribution function. The second curve in Fig. 4 demonstrates the deformation of the energetic particle distribution during the formation of the cliff $\left(\varphi_{2} \approx 0.07^{\circ}\right)$, the third curve corresponds to the maximum value of the trapped electrons flux at low heights $\left(\varphi_{3} \approx 0.1^{\circ}\right)$, and the last curve presents the relaxation phase $\left(\varphi_{4} \approx 0.62^{\circ}\right)$. In the $\mathrm{SD}$ case the distribution function relaxes to an isotropic distribution, which corresponds to the linear dependence on $b$ [Eq. (54)] given in Fig. 4, curves 3 and 4. In Fig. 5 the second

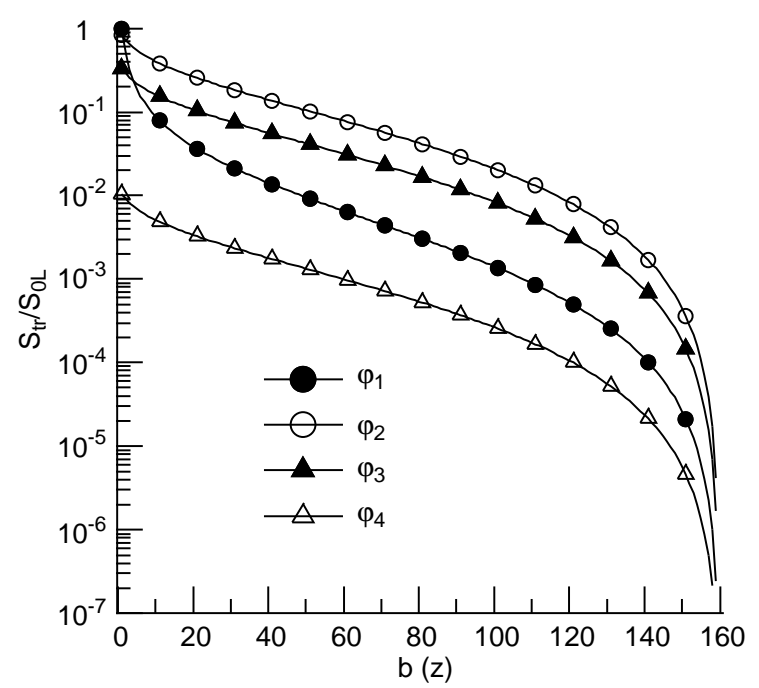

Fig. 5. The dependence of fluxes of trapped electrons along a magnetic flux tube on $b(z)=B(z) / B_{L}$ in the case of weak diffusion; $\mu_{c}^{-1}=160, \quad D_{m} \mu_{c}^{-1} \approx 0.3 \quad\left(B_{1} \approx 0.5 \mathrm{~s}^{-1}, \quad\right.$ if $\left.\quad v_{g \perp} \approx 10^{2} \mathrm{~km} / \mathrm{s}\right)$, $\ln \left(D_{m} / D_{0}\right) \approx 10, \quad v_{0} / B_{1}=0.01, \quad \varphi_{1}=0, \quad \varphi_{2} \approx 4.2^{\circ}, \quad \varphi_{3} \approx 7^{\circ}$, $\varphi_{4} \approx 14.1^{\circ}$ curve corresponds to the maximal value of the trapped electrons flux at low heights $\left(\varphi_{2} \approx 4.2^{\circ}\right)$, and the two other curves demonstrate the relaxation phase $\left(\varphi_{3} \approx 7^{\circ}\right.$, $\left.\varphi_{4} \approx 14.1^{\circ}\right)$. The parallel curves on the logarithmic scale (Fig. 5, curves 2, 3 and 4) show that in the WD case, the distribution approaches a general dependence on $\mu$ $\left(Z_{1}^{W}(\mu)\right)$ with decreasing amplitude, as $\varphi$ increases.

In both regimes we see a cliff-like deformation of the distribution function at the initial stage of relaxation $(\varphi \rightarrow 0)$, which takes place for sufficiently low heights $\left(h \sim 10^{3} \mathrm{~km}\right)$. All these features are in good accordance with NOAA satellite data (Yahnina et al., 1996). As a rule, energetic electrons observed by the NOAA satellites show a very sharp cliff and corresponding precipitation, with a typical front width $\Delta \varphi_{F} \sim 0.1^{\circ}-0.3^{\circ}$; these values are very similar to the theoretical results in the case of SD (see Fig. 2).

\section{Conclusion}

The theoretical model developed here is very useful for the explanation of the main features of energetic electron fluxes observed aboard low-altitude satellites as the energetic electrons drift into a region of enhanced plasma density and precipitate from the outer radiation belt. Our model can provide the basis for further, more sophisticated analyses of particle and wave data. The next experimental steps could be in the direction of a more precise analysis of the pitch-angle and energy dependences of an evolving distribution function along the satellite trajectory, as well as of amplitude and spectral features of ELF waves in particular data sets. These analyses would permit us to formulate stricter approaches for a theoretical model.

It is important to develop further the theoretical model of wave energy transfer equation. In the present model, wave propagation across the magnetic field was included with a free parameter, the group velocity component $v_{g \perp}$. This parameter determines important features of the solution and, of course, demands further self-consistent consideration, with peculiarities of the whistler wave propagation near the plasmapause being taken into account.

Finally, a very important question is whether the time-stationary solution of the system of equations given by Eq. (18) is appropriate for the magnetosphere. Many experimental data and some theoretical models demonstrate not a stationary but a burst-like behaviour of the cyclotron instability in the magnetosphere, the strongest evidence being that of ELF-VLF chorus emissions. It is therefore necessary to develop nonstationary models of cyclotron wave-particle interactions. There have already been some successful attempts in this direction, an example being that of pulsating aurora (Demekhov and Trakhtengerts, 1994).

Acknowledgements. This work was supported in part by INTAS, grant 94-2753, and by the Russian Foundation for Basic Research, grant 96-02-16473a.

Topical Editor K.-H. Glapmeier thanks D. Nunn and another referee for their help in evaluating this paper. 


\section{References}

Bespalov, P. A., and V. Y. Trakhtengerts, The cyclotron instability in the Earth radiation belts. In Reviews of plasma physics, vol. 10, Ed. M. A. Leontovich, Plenum, New York, pp. 155-192, 1986.

Bespalov, P. A., A. Grafe, A. G. Demekhov, and V. Y. Trakhtengerts, Some aspects of the asymmetric ring current dynamics, Geomagn. Aeron., 30, 740-746, 1990.

Bespalov, P. A., A. Grafe, A. G. Demekhov, and V. Y. Trakhtengerts, On the role of collective interactions in asymmetric ring current formation, Ann. Geophysicae., 12, 422-430, 1994.

Demekhov, A. G., and V. Y. Trakhtengerts, A mechanism of formation of pulsating aurorae, J. Geophys. Res., 99, 5831$5841,1994$.

Kennel, C. F., Consequences of a magnetospheric plasma, Rev. Geophys., 7, 339-419, 1969.

Korn, G., and T. Korn, Mathematical handbook for scientists and engineers, McGraw-Hill, New York, 1961.

Lyons, L. R., and D. J. Williams, Quantitative aspects of magnetospheric physics, Reidel, Dordrecht, 1984.

Semenova, V. I., and V. Y. Trakhtengerts, On some peculiarities of waveguide propagation of low-frequency waves in magnetosphere, Geomagn. Aeron., 10, 1021-1027, 1980.
Strangeways, H. J., The upper cut-off frequency of nose whistlers and implications for duct structure, J. Atmos. Terr. Phys., 53, 151-169, 1991.

Titova, E. E., T. A. Yahnina, A. G. Yahnin, B. B. Gvozdevsky, A. A. Lyubchich, V. Y. Trakhtengerts, A. G. Demekhov, J. L. Horwitz, F. Lefeuvre, D. Lagoutte, J. Manninen, and T. Turunen, Strong localized variations of the low-altitude energetic electron fluxes in the evening sector near plasmapause, Ann. Geophysicae, in press, 1997.

Trakhtengerts, V. Y., A. A. Lyubchich, A. G. Demekhov, T. A. Yahnina, E. E. Titova, M. J. Rycroft, J. Manninen, and T. Turunen, Cyclotron model for quasi-steady precipitation of energetic electrons at the plasmapause. In Proc. XIX seminar on auroral phenomena, Apatity, Russia, PGI, pp. 73-76, 1996.

Yahnina, T. A., E. E. Titova, A. G. Yahnin, B. B. Gvozdevsky, A. A. Lyubchich, V. Y. Trakhtengerts, A. G. Demekhov, J. L. Horwitz, J. Manninen, and T. Turunen, Some features in the energetic electron precipitation pattern near the plasmapause in the evening sector. In Proc. XIX seminar on auroral phenomena, Apatity, Russia, PGI, pp. 70-72, 1996. 